\title{
Annual reports on hypertension research 2020
}

\author{
Masaki Mogi ${ }^{1} \cdot$ Yukihito Higashi $^{2,3} \cdot \mathrm{Kanako} \mathrm{Bokuda}^{4} \cdot$ Atsuhiro Ichihara $^{4} \cdot$ Daisuke Nagata $^{5} \cdot$ Atsushi Tanaka $^{6}$. \\ Koichi Node ${ }^{6}$. Yoichi Nozato ${ }^{7}$ Koichi Yamamoto ${ }^{7} \cdot$ Ken Sugimoto $^{8} \cdot$ Hirotaka Shibata $^{9} \cdot$ Satoshi Hoshide $^{10}$. \\ Hitoshi Nishizawa ${ }^{11} \cdot K^{\prime} a z u o m i ~ K a r i o{ }^{10}$
}

Received: 13 September 2021 / Accepted: 14 September 2021 / Published online: 15 October 2021

(c) The Japanese Society of Hypertension 2021

\begin{abstract}
In 2020, 199 papers were published in Hypertension Research. Many excellent papers have contributed to progress in research on hypertension. Here, our editorial members have summarized eleven topics from published work and discussed current topics in depth. We hope you enjoy our special feature, Annual Reports on Hypertension Research.
\end{abstract}

Keywords Hypertension Research • Annual topics • Publication in 2020

\section{Hot topics on vascular function in hypertension, 2020}

\section{(See Supplementary Information 1)}

Several methods for assessment of vascular function including endothelial function, vascular smooth muscle function, arterial stiffness, and biochemical markers have been used in patients with hypertension (Fig. 1) [1-7]. These methods have been established as markers for the grade of atherosclerosis, markers for efficacy of interventions, tools for evaluating mechanisms of atherosclerosis,

Supplementary information The online version contains supplementary material available at https://doi.org/10.1038/s41440021-00766-3.

Masaki Mogi

mmogi@m.ehime-u.ac.jp

1 Deparment of Pharmacology, Ehime University Graduate School of Medicine, Tohon, Ehime, Japan

2 Department of Cardiovascular Regeneration and Medicine, Research Institute for Radiation Biology and Medicine, Hiroshima University, Hiroshima, Hiroshima, Japan

3 Divivsion of Regeneration and Medicine, Medical Center for Translational and Clinical Research, Hiroshima University Hospital, Hiroshima, Hiroshima, Japan

4 Department of Endocrinology and Hypertension, Tokyo Women's Medical University, Shinjuku, Tokyo, Japan

5 Division of Nephrology, Department of Medicine, Jichi Medical University School of Medicine, Shimotsuke, Tochigi, Japan and predictors of cardiovascular outcomes in cardiovascular disease (CVD) including hypertension (Fig. 1) [8-12].

There were many reports on "vascular function and hypertension" in Hypertension Research in 2020 alone. Liu et al. [13] demonstrated a significant association of higher brachialankle pulse wave velocity (baPWV) as an index of arterial stiffness with a higher frequency of the presence of carotid plaque in patients with hypertension, particularly in relatively young patients. Maruhashi et al. [14] showed that measurement of baPWV is a predictor of cardiovascular events in hypertensive patients with coronary artery disease with controlled blood pressure (BP) of $<130 / 80 \mathrm{mmHg}$, suggesting that a $\mathrm{BP}$ target of $<130 / 80 \mathrm{mmHg}$ is appropriate for the treatment of hypertensive patients with coronary artery disease. Tomiyama et al. (Supplementary Information 1-1) showed that the SAGE score calculated from age, office systolic BP, fasting blood

6 Department of Cardiovascular Medicine, Saga University, Saga, Saga, Japan

7 Department of Geriatric and General Medicine, Osaka University Graduate School of Medicine, Suita, Osaka, Japan

8 General and Geriatric Medicine, Kawasaki Medical University, Okayama, Okayama, Japan

9 Department of Endocrinology, Metabolism, Rheumatology and Nephrology, Faculty of Medicine, Oita University, Yufu, Oita, Japan

10 Division of Cardiovascular Medicine, Department of Medicine, Jichi Medical University School of Medicine, Shimotsuke, Tochigi, Japan

11 Department of Metabolic Medicine, Osaka University Graduate School of Medicine, Suita, Osaka, Japan 
Fig. 1 Methods for assessment of vascular function (top) and clinical efficacy of assessment of vascular function (bottom). FMD indicates flow-mediated vasodilation, ezFMD enclosedzone FMD, RHI reactive hyperemia index, NID nitroglycerine-induced vasodilation, baPWV brachialankle pulse wave velocity, cfPWV carotid-femoral PWV, CAVI cardio-ankle vascular index, AI augmentation index, TBI toe brachial pressure index, ABI ankle-brachial pressure index, IMT intima media thickness
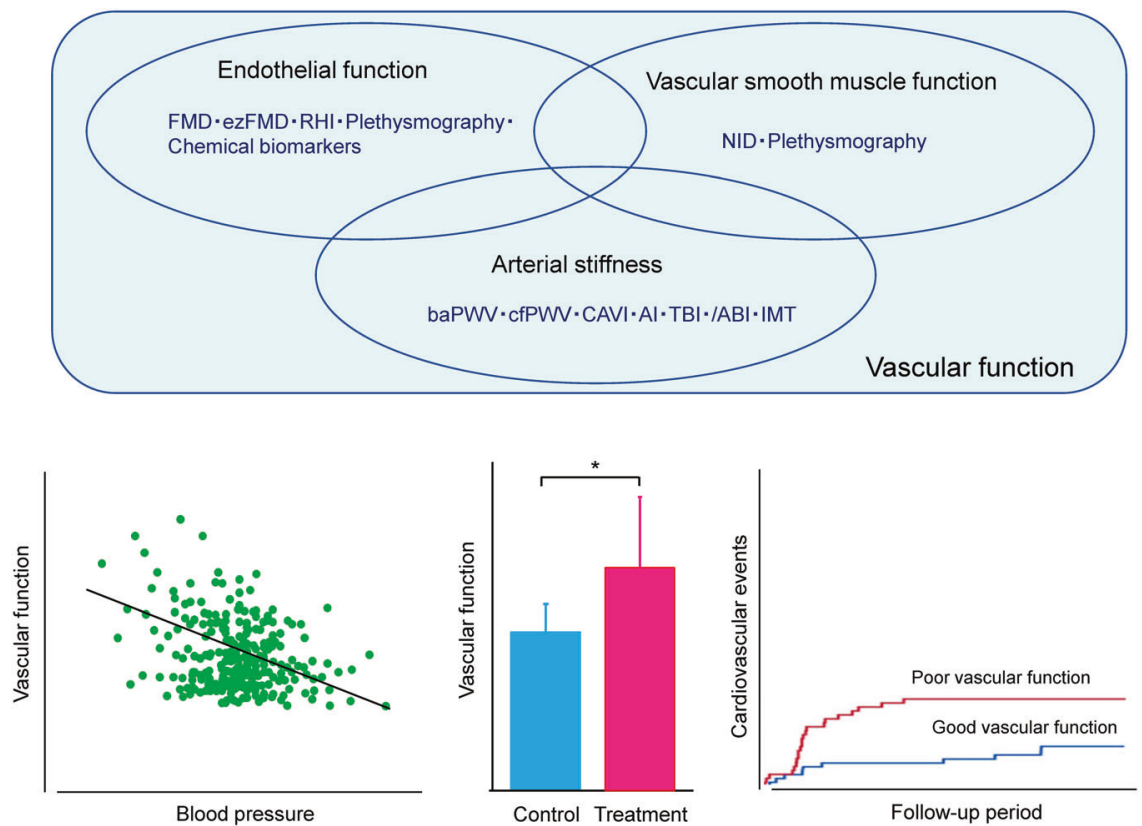

Follow-up period glucose, and estimated glomerular filtration rate (eGFR) is useful for predicting baPWV in patients with hypertension. In addition, elevation of BP contributes, at least in part, to microvascular dysfunction (Supplementary Information 1-2). The association between quality of sleep and vascular function in patients with hypertension remains unclear. Hu et al. (Supplementary Information 1-3) reported that poor sleep quality was associated with higher arterial stiffness measured by baPWV in patients with hypertension. It has also been reported that measurement of carotid-femoral PWV reveals different hypertension phenotypes including white-coat hypertension, resistant hypertension and nonresistant hypertension (Supplementary Information 1-4). Interestingly, it has been shown that high BP-induced alteration of shear stress reduces antithrombotic effects through a decrease in nitric oxide bioavailability in human conduit arteries, leading to an increase in risk of arterial thrombosis [15].

In fields other than hypertension, Fukumoto et al. (Supplementary Information 1-5) showed that smoking cessation improved flow-mediated vasodilation as an index of macrovascular endothelial function but not reactive hyperemic index as an index of microvascular endothelial function, suggesting that attention should be paid to the effects of smoking on endothelial function in vessels of different sizes. Maruhashi et al. [16] confirmed that vascular function, including endothelial function, vascular smooth muscle function and arterial stiffness, is continuously impaired throughout life with aging. Harada et al. (Supplementary Information 1-6) showed that vascular dysfunction plays a critical role in the decrease in skeletal muscle mass, leading to cardiovascular events. Also, microcirculatory dysfunction assessed by digital capillary density and high normal anklebrachial pressure index independently contributes to advancement of chronic kidney disease stages (Supplementary Information 1-7, 1-8). The advanced glycation end products (AGEs)/soluble receptor for AGEs pathway is associated with arterial stiffness in the general population (Supplementary Information 1-9).

Next year and also in the next decade, we would like to pay attention to publications concerning "vascular function and hypertension" in Hypertension Research. Accumulation of data on vascular function in hypertension would enable more specific conclusions concerning the roles of vascular function in the pathophysiology, maintenance, and development of hypertension to be drawn.

\section{Preeclampsia update}

\section{(See Supplementary Information 2)}

No effective treatment that attenuates the progression of preeclampsia (PE) has been established. New trials and cohort studies have provided insights into the development of preventive care and diagnostic and prognostic tools, and reports regarding these issues have also been published in Hypertension Research.

\section{Pathogenesis}

The two-stage paradigm of poor early placental development followed by systemic endothelial dysfunction and severe maternal organ injury is an effective model to frame the pathogenesis of PE (Fig. 2) [17]. Vangrieken et al. (Supplementary Information 2-1) investigated the effect of placental hypoxic-conditioned medium on intraluminalinduced contraction and endothelial barrier integrity in 
Fig. 2 Topics on preeclampsia, 2020. PE preeclampsia, BP blood pressure, sFlt-1 soluble fms-like tyrosine kinase-1, sENG soluble endoglin, PRES posterior reversible encephalopathy syndrome, AKI acute kidney injury, CsA cyclosporin A, CKD chronic kidney disease

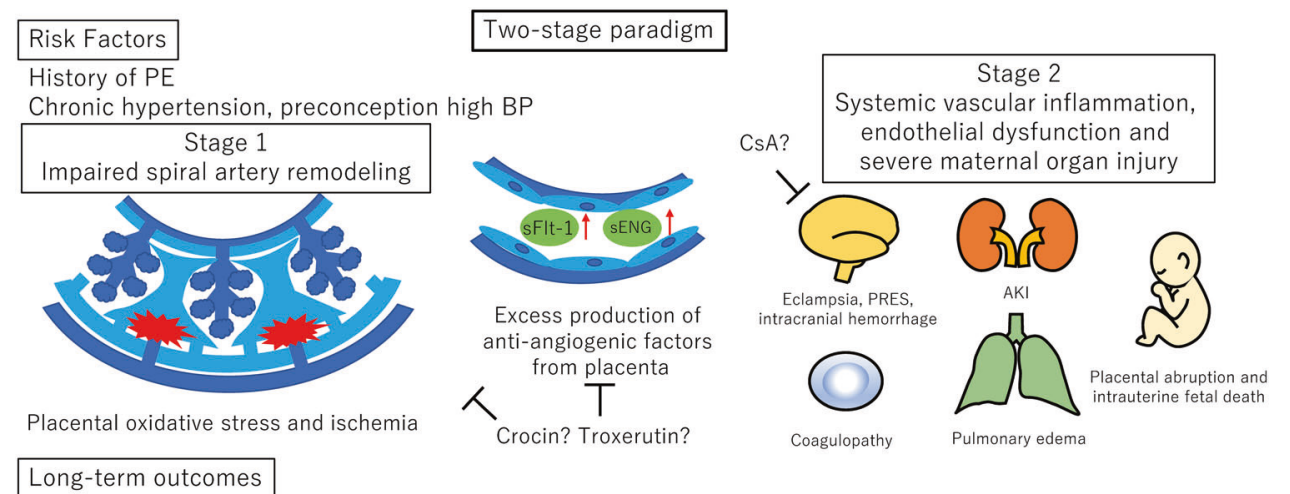

Hypertension, cardiovascular disease, diabetes, CKD, dementia chorionic arteries, and have demonstrated a link between factors secreted by placental cells in response to hypoxia and vascular abnormalities.

\section{Preventive care}

The strongest risk factors for PE are history of PE and chronic hypertension. Li et al. showed associations between high preconception BP level and an increase in risk of gestational hypertension and $\mathrm{PE}$, indicating the importance of preconception BP control. Some risk factors are amendable to prepregnancy modifications [18]. Li et al. (Supplementary Information 2-2) demonstrated that different subclasses of saturated fatty acids show diverse effects on the risk of pregnancyinduced hypertension, and suggested that dietary saturated fatty acids may be a novel means by which to decrease BP during pregnancy.

\section{Diagnostic and prognostic tools}

To make an early prediction of PE before diagnosis, Yue et al. (Supplementary Information 2-3) developed a nomogram that incorporates BMI, BP, uterine artery ultrasound parameters, and serological indicators for the early prediction of $\mathrm{PE}$ in pregnant Chinese women. Also, recently, the Fetal Medicine Foundation proposed a Bayes theorem-based model to predict preterm PE. Goto et al. investigated the diagnostic accuracy of the model and showed that the model is feasible in the Japanese population [19].

\section{Management}

Once diagnosed, PE is often a progressive condition and maternal organ function deteriorates with time. Chen et al. (Supplementary Information 2-4) have reported that crocin alleviates inflammatory and oxidative stress in placental tissues, thereby protecting against gestational hypertension (Fig. 2). Li et al. (Supplementary Information 2-5) explored the efficacy of troxerutin and found that it reduces BP and the expression of vasodilation converting enzyme, angiotensin, urinary protein, and pro-inflammatory cytokines while increasing the expression of anti-inflammatory cytokines (Fig. 2).

Magnesium sulfate reduces the risk of an eclamptic seizure, but its mechanism of action is poorly understood. Li et al. (Supplementary Information 2-6) have shown that prophylactic magnesium sulfate decreases BP and attenuates the postpartum effects of PE. Huang et al. (Supplementary Information 2-7) have shown that the maternal hyperinflammatory response in PE lowers the eclampsia-like seizure threshold [20] and found that administration of a well-known immunosuppressive agent, cyclosporin A (CsA), effectively attenuated PE manifestation, and eclampsia-like seizure severity and improved subsequent pregnancy outcomes in a rat model (Fig. 2).

\section{Long-term outcomes}

PE confers an increased risk of major chronic diseases in later life including many cardiovascular complications [21], diabetes [22], chronic kidney disease [23], and also dementia [24]. Wagata et al. have shown that the association between hypertensive disorders in pregnancy (HDP) and later hypertension was stronger in younger women, implying that continuous follow-up after the postpartum period is important for women with a history of HDP for early detection of hypertension in later life [25].

The 2019 Maternal Mortality update from the WHO report indicated a major contribution of $\mathrm{PE}$ and eclampsia to worldwide maternal deaths. Much work is needed to decrease its morbidity and mortality, and this may be reported in Hypertension Research in the future.

\section{Treatment of hypertensive patients during COVID-19 pandemic}

(See Supplementary Information 3)

During 2020, the coronavirus disease 2019 (COVID-19) pandemic has been the most defining global health crisis 


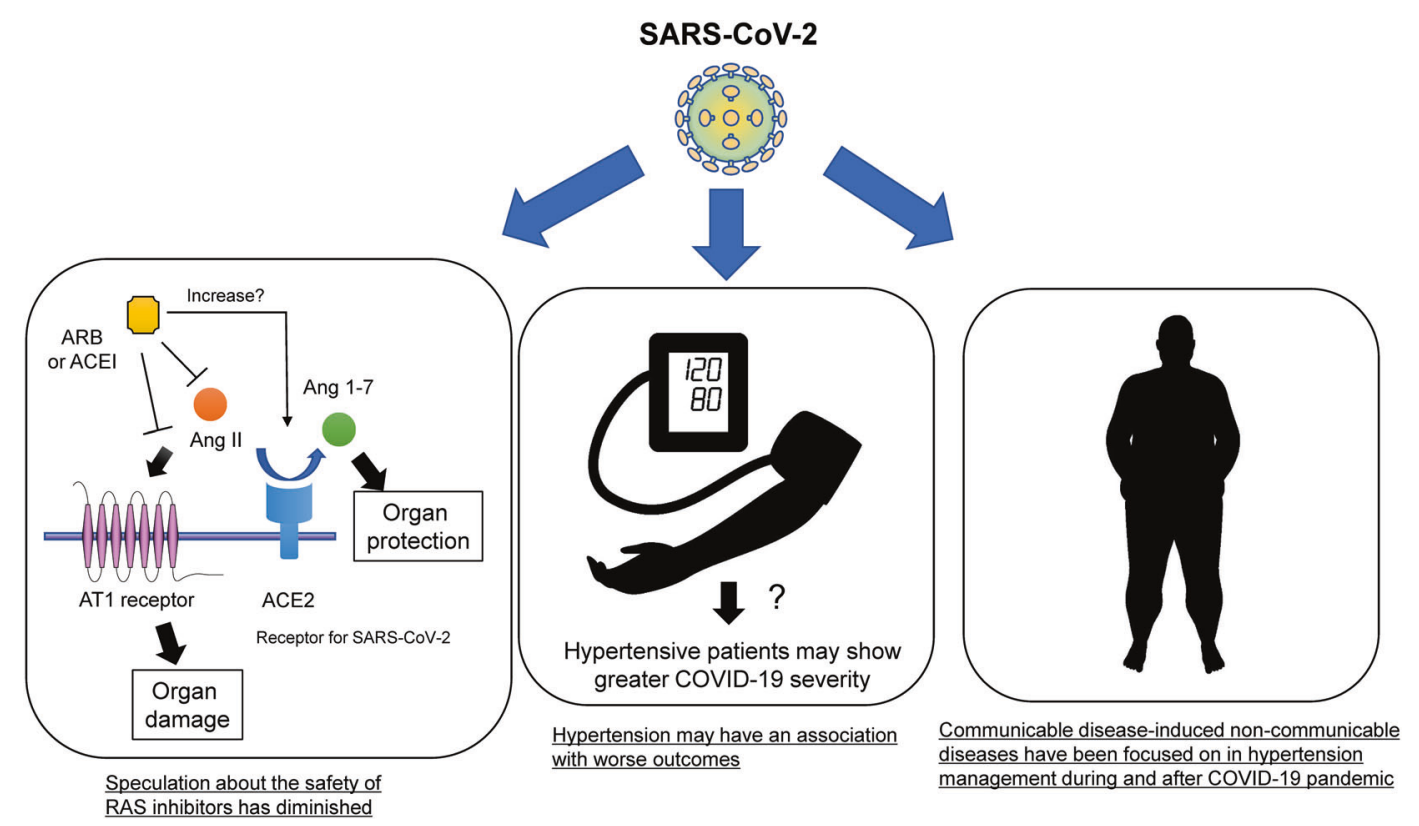

Fig. 3 Topics on COVID-19

worldwide. Many reports related to this international public health emergency have been published in Hypertension Research. Presidents of the Japanese Society of Hypertension (JSH) have written communications about proposals for future hypertension treatment, conscious of the "new normal" during and after COVID-19. Shibata and JSH members also strived together to summarize and discuss the effects and severity of severe acute respiratory syndrome coronavirus 2 (SARS-CoV-2) infection and the effects of antihypertensive drugs, especially in relation to blockade of the renin-angiotensin system (RAS) [26]. The most downloaded article in 2020 was a short communication by $\mathrm{Li}$ et al. (Supplementary information 3-1), demonstrating the association between COVID-19 and RAS inhibitors. On March 4, the date on which the communication was accepted, there was concern that ACE2, which is considered to be upregulated by RAS inhibitors, could promote the proliferation of COVID-19 and enhance its capability for infection. Kai et al. (Supplementary Information 3-2) promptly reviewed the interactions between coronaviruses and ACE2, angiotensin II, and RAS inhibitors. This review was the second most downloaded article in 2020. Furuhashi et al. (Supplementary Information 3-3) also commented on this topic around the same time. To address this question, Matsuzawa et al. clearly demonstrated that RAS inhibitors could be beneficial for the prevention of confusion in COVID-19 patients with hypertension [27]. Following assessment of the clinical studies, speculation about the safety of RAS inhibitors has diminished [28, 29] (Fig. 3).

The next hot topic is the effect of hypertension and BP control on COVID-19 severity. Huang et al. (Supplmentary Information 3-4) showed that COVID-19 patients with hypertension were more likely to have severe pneumonia and excessive inflammatory reactions compared with patients without hypertension. Ran et al. (Supplmentary Information 3-5) also showed that BP control is independently associated with mortality and intensive care unit (ICU) admission. Hypertension has been identified as the most common comorbidity in COVID-19 patients and has shown an association with worse outcomes, possibly due to more advanced atherosclerosis and target organ damage, in a recent report [30]. More attention should be paid to avoiding SARS-CoV-2 infection in hypertensive patients in order to prevent severe COVID-19 disease (Fig. 3).

Finally, the COVID-19 pandemic has changed lifestyle factors and behaviors such as physical activity, dietary pattern, alcohol consumption and mental conditions, resulting in an increase in metabolic syndrome with body weight gain. These communicable disease-induced noncommunicable diseases [31-33] have been focused on in hypertension management during and after the COVID-19 pandemic and may be reported in Hypertension Research in the future (Fig. 3).

\section{Treatment of CKD patients and therapeutic usefulness of SGLT2 inhibitors}

(See Supplementary Information 4)

The Kidney Disease: Improving Global Outcomes (KDIGO) 2021 Clinical Practice Guidelines for the Management of BP in Chronic Kidney Disease (CKD) [34] represents an update to the KDIGO 2012 guidelines. The updated guidelines include a chapter dedicated to 
Fig. 4 Mechanisms by which SGLT2 inhibitors might improve cardiac and renal function

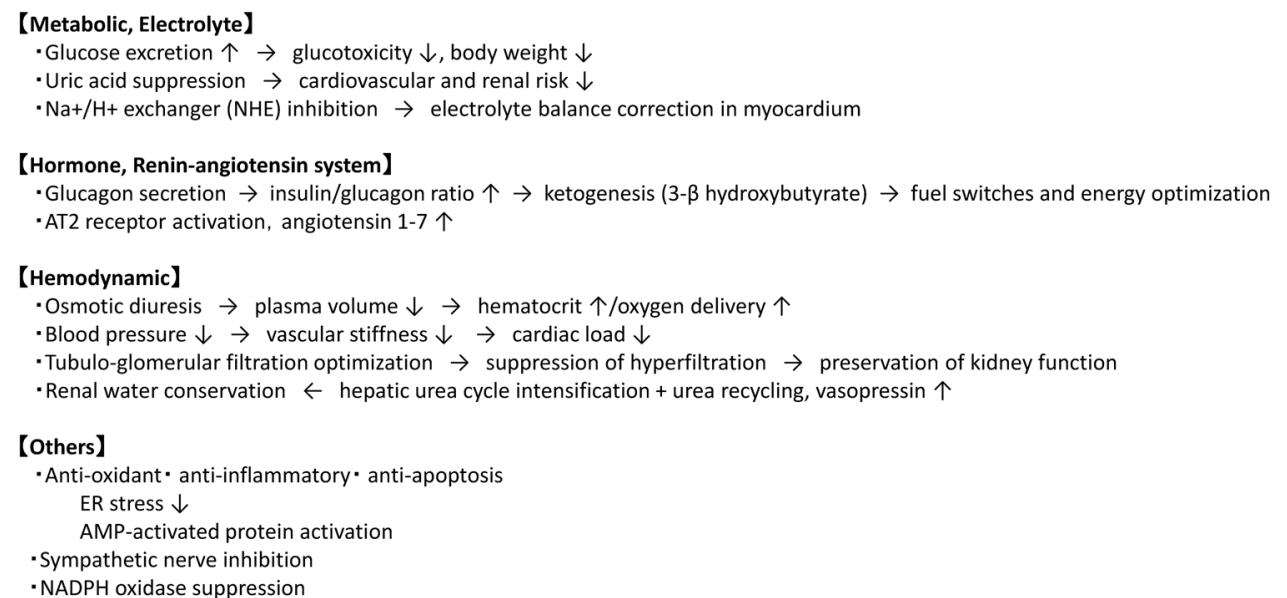

standardized office BP (OBP) measurement for most patients with CKD, who do not undergo dialysis. With regard to recommendations for standardized OBP measurements, based on previous and new evidence, particularly the results of the Systolic Blood Pressure Intervention Trial (SPRINT) [35], these guidelines propose a systolic BP target of $<120 \mathrm{mmHg}$. Renin-angiotensin system inhibitors (RASis) are recommended as first-line medication for patients with high BP and CKD accompanied by proteinuria. Although these guidelines include advice regarding salt reduction, reduction of salt intake is difficult in realworld practice. Yoshimura et al. [36] reported that excessive alcohol consumption enhanced the effect of salt intake on albuminuria. The benefit of salt restriction in reducing albuminuria is greater in patients who successfully limit alcohol consumption. Data regarding the addition of mineralocorticoid receptor antagonists (MRA) to RASis are unavailable in the headlines of the updated guidelines. However, finerenone (a selective MRA) was shown to significantly reduce the risk of CKD progression and cardiovascular events in patients with CKD with concomitant type 2 diabetes. [37] Wang et al. (Supplementary Information 4-1) reported that an appropriate dose of spironolactone may improve endothelial function, as reflected by a lower prevalence of microalbuminuria in patients with primary aldosteronism, which suggests that in addition to their beneficial effects on the endothelium, MRAs may reinforce the cardio-renal protective effects of RASis. Zamami et al. (Supplementary Information 4-2) proposed the application of the ankle-brachial index (ABI) as a useful tool for evaluation of CKD severity. ABI was positively correlated with biopsy-proven severity of renal small artery intimal thickening in patients with $\mathrm{CKD}$, which indicates that an elevated ABI may predict renal small artery remodeling and a low eGFR. Based on quantitative nailfold video capillaroscopy assessment, Schoina et al. (Supplementary Information 4-3) reported that capillary density progressively decreased with advancing CKD stage. The authors observed structural alterations in the microvasculature in parallel to arteriosclerosis in large and medium-sized arteries in patients with CKD, which supports the usefulness of capillary density as a predictor of CKD severity and the risk of cardiovascular events.

Independent of diabetes, sodium-glucose cotransporter-2 inhibitors (SGLT2is) have gained much attention in the field of nephrology in recent times. Although SGLT2is were originally introduced as antidiabetic agents, several studies have shown that these drugs improved cardiovascular and renal prognosis in patients with CKD [38-44]. The mechanisms underlying SGLT2i activity include selective inhibition of SGLT2 expressed in the S1 segment of the proximal tubule, promotion of hypotensive activity, and improvement of glucose metabolism. SGLT2is also increase the quantity of sodium chloride delivered to the macula densa of the distal tubule and correct glomerular hyperfiltration by promoting contraction of the afferent arteriole via the tubuloglomerular feedback mechanism, which serves as a major contributor to the renoprotective effects of SGLT2is (Fig. 4) [45]. Furthermore, SGLT2is reduce adverse left ventricular remodeling and improve systolic function by switching cardiac metabolism from glucose consumption toward utilization of more energyproducing ketone bodies, free fatty acids, and branchedchain amino acids, which results in improved myocardial energetics and efficiency [46, 47]. SGLT2i-induced sympathetic nerve inhibition was shown to be a critical contributor to the cardio-renal protective effects associated with SGLT2is in some nondiabetic animal models [48, 49]. Compared with RASi monotherapy, combination therapy using SGLT2is and RASis was effective and well tolerated in patients with diabetes and could improve renal outcomes. However, this therapeutic approach was associated with an increased risk of hypoglycemia [50]. Kitamura et al. [51] reported that pre-treatment administration of metformin 


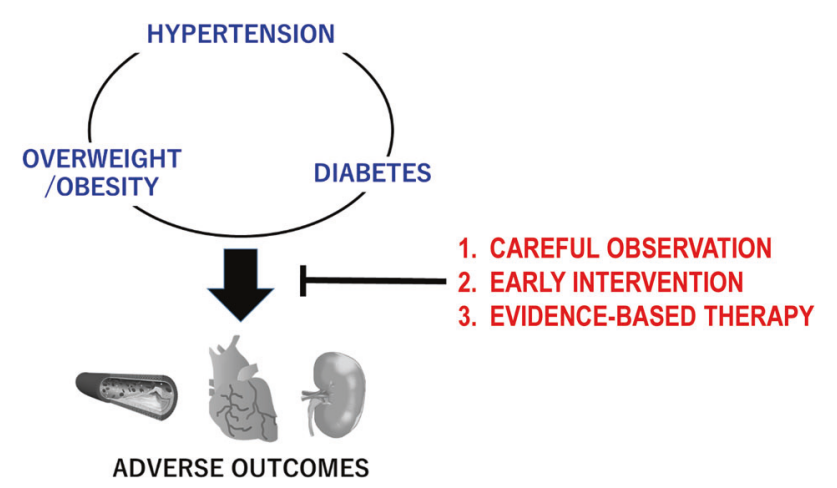

Fig. 5 Topics on obesity/diabetes

diminished the SGLT2i-induced reduction in eGFR, and addition of RASi therapy restored this response. This study highlights that SGLT2i-induced renal microvascular action may be mediated predominantly by post-glomerular vasodilatation as opposed to pre-glomerular vasoconstriction, which has been considered the primary underlying mechanism. Finally, the initial decrease in eGFR during the early stages of SGLT2 $i$ therapy was a concern expressed by several nephrologists, and Kohagura et al. (Supplementary Information 4-4) showed that eGFR measured during the chronic phase after luseogliflozin administration remained stable regardless of the severity of the initial acute changes.

\section{Obesity/diabetes}

\section{(See Supplementary Information 5)}

Under the COVID-19 catastrophe, many people are likely forced to face various health problems caused by disordered eating habits and physical inactivity, leading to overweight/obesity, hypertension, and metabolic syndrome. At the same time, the baseline obesity/overweight and impaired metabolic conditions are also associated with adverse outcomes in patients infected with COVID-19 [5254]. There is now a renewed need to focus on the clinical management of obesity/overweight and its association with hypertension (Fig. 5). Thu et al. (Supplementary Information 5-1) demonstrated a strong association between the burden of visceral adipose tissue and systolic BP, independent of serum levels of inflammatory markers, in midlife Singaporean women. Intriguingly, among Japanese women, a history of hypertensive disorders of pregnancy was significantly associated with later development of hypertension even in younger generations, and overweight/obesity unfavorably enhanced that association [25]. Thus, it is obvious that overweight/obesity incrementally increases the level of BP and accelerates the risk of hypertension; this fact no longer needs a reference. To adjust or maintain an individual's ideal body weight, it also goes without saying that comprehensive lifestyle modification, including exercise and diet, is essential. Regarding exercise-training, Clark et al. sought to determine an optimal practical program for reducing BP in men with overweight/obesity. They found that a series of high-intensity interval training was more effective for central and peripheral BP-lowering than was moderate-intensity continuous training, and those effects were more pronounced in overweight/obese subjects with higher baseline BP [55]. On the other hand, a higher frequency of alcohol consumption, which is also a current socio-physical problem, was associated with higher incidence of hypertension in nonobese men, but not women and overweight/obese men [56]. Importantly, although the relationship between alcohol intake and the development of hypertension and cardiovascular events is not necessarily a simple one [57-59], it is best not to drink, or for habitual drinkers to try to stay sober regardless of overweight/obesity and sex.

Type 2 diabetes (T2D) and hypertension synergistically increase the risk of cardiovascular events and mortality. Imai et al. investigated the impact of hypertension stratified by diabetes status on the lifetime risk of cardiovascular death using a Japanese cohort of the EPOCH-JAPAN study. They found that hypertension and diabetes were associated with a markedly increased lifetime risk in a synergistic manner even at an earlier index age [60], suggesting the importance of social awareness and prevention of these factors from the younger generation. Thus, constant BP measurement/monitoring and comprehensive clinical management of relevant risk factors, including hypertension, are of importance especially in patients with T2D. Ushigome et al. examined the predictive impact of BP values measured at home on the first onset of major adverse cardiovascular events in Japanese patients with T2D with no previous history of CVD (KAMOGAWA-HBP study). They reported that a rise in morning systolic BP by $10 \mathrm{mmHg}$ was independently associated with an increased risk of development of cardiovascular events (adjusted hazard ratio [HR] $1.14,95 \%$ confidence interval 1.01 to 1.28 ), and HR in patients with morning systolic $\mathrm{BP} \geq 135 \mathrm{mmHg}$ was numerically larger than that in those with morning systolic $\mathrm{BP}<125 \mathrm{mmHg}$ [61]. However, it has long been controversial whether lower BP is associated with better outcomes in patients with T2D, although BP-lowering of 10 $\mathrm{mmHg}$ was associated with apparent clinical benefits in patients with $\mathrm{T} 2 \mathrm{D}$ and baseline $\mathrm{BP} \geq 140 \mathrm{mmHg}$ [62]. On the basis of previous meta-analyses and the expected benefit on the risk of stroke in the Japanese population [63-65], the current JSH guidelines recommend a BP of less than 130/ $80 \mathrm{mmHg}$ as a treatment target for OBP in patients with T2D complicated with hypertension [66]. Generally, in patients with hypertension complicated with atherosclerotic cardiovascular disease (ASCVD), careful attention should 
Fig. 6 Advantages of home BP monitoring

\section{Advantages of home BP monitoring}

Evaluation of BP values $\quad[78,79,80$, Suppl.6-1,2]

- Diagnosis of white-coat and masked hypertension

- Diagnosis of resistant hypertension

- Detection of BP variability

\section{Assessment of cardiovascular risk [Suppl.6-3,4,5]}

- Prognostic factors for cardiovascular events and mortality

Treatment of hypertension [81,Suppl.6-6,7,8,9]

- Selection of appropriate antihypertensive medication

- Patient education (awareness of disease, encouraging change in behavior, improving medication adherence)

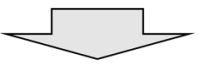

Improve quality of hypertension management (BP lowering and reduction of cardiovascular events or mortality) be paid to the decrease in organ perfusion associated with BP-lowering. Kai et al. examined whether lower BP levels independently contributed to the incidence of cardiovascular events in patients with T2D who underwent coronary revascularization (CREDO-Kyoto cohort-1 registry). They demonstrated that clinically-allowable levels of low BP were not associated with an increased risk of ASCVD and mortality [67], supporting the current clinical practice of BP-lowering in patients with T2D recommended by the JSH 2019 guidelines [66].

Finally, we would like to briefly introduce some exciting progress in exploratory and basic research in the area of "Obesity/Diabetes". Gelžinský et al. investigated how to better depict the burden of advanced glycation end products (AGEs) related to arterial stiffness. They revealed that skin AGEs quantified by a noninvasive autofluorescence device and its ratio to the circulating concentration of soluble receptor for AGEs were strongly and independently associated with increased arterial stiffness assessed as aortic pulse wave velocity [68], suggesting that this simple method is clinically useful to assess "vascular age" and/or "vascular failure" [69]. Hirao et al. examined the effect of pharmacological blockade of vacuolar type $\mathrm{H}^{+}$-ATPase (VATPase) in a rat model of T2D. They newly found that blockade of V-ATPase by bafilomycin inhibited renal ammoniagenesis and gluconeogenesis and improved pancreatic insulin secretion and its sensitivity [70], shedding light on the pathophysiological role of V-ATPase as a novel therapeutic target in T2D. Heart failure is a common complication in patients with T2D, and this pathological condition requires novel therapeutic approaches beyond glucose-lowering therapy [71]. Wang et al. focused on the role of translocase of mitochondrial outer membrane 70 (Tom70) in the pathogenesis of diabetic cardiomyopathy. They first revealed that genetic rescue of Tom70 expression protected against mitochondrial dysfunction and improved the cardiac phenotype of diabetic cardiomyopathy in $\mathrm{db} / \mathrm{db}$ mice [72], indicating that Tom70 may become an emerging therapeutic target specifically for diabetic cardiomyopathy.

\section{Hypertension management}

(See Supplementary Information 6)

Given the importance of intensive BP treatment implicated in recent clinical trials including SPRINT [73], it is increasingly important to clarify how hypertensive patients need to be managed in clinical practice. In this context, recent studies have focused on the appropriate use and interpretation of home BP (HBP) [74] including selfmeasured BP (SMBP) and ambulatory BP (ABP), which enables us to detect a high-risk population and reduce cardiovascular risk [75-77]. We reviewed studies related to HBP and hypertension management that were published on hypertension research in 2020. The subjects of these studies can be categorized into "evaluation of BP values", "assessment of cardiovascular risk", and "treatment of hypertension” (Fig. 6).

Regarding the evaluation of BP values, Kubozono et al. (Supplementary Information 6-1) demonstrated that HBP was significantly correlated with room temperature. Asayama et al. (Supplementary Information 6-2) revealed the current status of HBP and home pulse rate in relation to sex, age, and antihypertensive treatment status in the Japanese general population. Kadowaki et al. demonstrated that OBP measured with a sufficient rest time (st-OBP) was comparable to HBP at the population level [78]. Interestingly, smokers and obese men taking antihypertensive drugs had higher HBP than st-OBP, indicating that their BP levels are at risk of being underestimated. Gkaliagkousi et al. reported a novel method to detect 
masked hypertension in individuals with normal OBP using a combination of conventional brachial BP and aortic BP (central BP) measured in the office [79]. Sheng et al. shed light on the pathophysiology of BP and pulse variability in relation to the autonomic nervous system, and demonstrated that individuals with masked hypertension showed variability in BP and heart rate, and baroreflex sensitivity similar to that in those with sustained hypertension [80].

Regarding the assessment of cardiovascular risk, Ushigome et al. (Supplementary Information 6-3) demonstrated that elevated morning BP is a future risk for cardiovascular events in a Japanese retrospective cohort study of type 2 diabetic patients. Manousopoulos et al. (Supplementary Information 6-4) showed that ABP variability was positively associated with LVMI in CKD patients. Ramoshaba et al. (Supplementary Information 6-5) demonstrated that masked hypertension was associated with retinal arteriolar narrowing in young healthy adults.

Regarding the treatment of hypertension, HBP monitoring has a beneficial effect on adherence and hypertension education. Zhang et al. reported that HBP measurement improved adherence and OBP control in a randomized controlled trial in Chinese subjects with stage 2 and 3 hypertension [81].

Vallée et al. (Supplementary Information 6-6) reported factors that improved adherence to antihypertensive treatment in a cross-sectional study in the French general population including elderly individuals. Nishimura et al. (Supplementary Information 6-7) revealed that although adherence was relatively high compared with that in Western countries, younger age, female sex, antihypertensive drug class, comorbid conditions, and types of medical institutions were associated with relatively low adherence. Nishigaki et al. (Supplementary Information 6-8) and Yoshida et al. (Supplementary Information 6-9) examined the gaps in perspectives on hypertension management between patients and physicians in the same panel-based, cross-sectional, observational study in Japan, and proposed a solution to the hypertension paradox. Nishigaki et al. demonstrated that perception of the amount of education provided by physicians on hypertension management was lower in patients than in physicians. Yoshida et al. demonstrated that, compared with specialist physicians, nonspecialists were less likely to provide adequate guidance on lifestyle modifications.

These reports support the importance of adequate HBP monitoring to improve hypertension management and reduce the risk of cardiovascular events and mortality.

\section{Hypertension and frailty}

(See Supplementary Information 7)

The relationship between frailty and hypertension has been studied. Guidelines for the treatment of hypertension in various countries show that older hypertensive patients with frailty, especially impaired physical function and/or activities of daily living (ADL), should be individually evaluated for the target $\mathrm{BP}$ and the initiation of antihypertensive medication $[66,82]$.

In Japan, the relationship between hypertension and frailty has been investigated mainly using cohort or crosssectional studies. Here, we introduce three papers published in Hypertension Research in 2020 and discuss the relationship between frailty and BP or cardiovascular risk.

In the SONIC study, an ongoing prospective cohort conducted since 2010, the relationship between systolic BP and physical frailty or cognitive function was examined in groups aged $70 \pm 1,80 \pm 1$, and $90 \pm 1$ years (Supplementary Information 7-1). Lower systolic BP was associated with a higher frequency of physical frailty in the 80-yearold group, but not in the 70- and 90-year-old groups, in subjects on antihypertensive medication; however, no association was found in the groups without antihypertensive medication. Besides, higher systolic BP was associated with lower cognitive function in the 70-year-old group, and lower systolic BP was associated with lower cognitive function in the 90 -year-old group in subjects on antihypertensive treatment; however, no association was found in the 80-year-old group on antihypertensive treatment or all the groups without antihypertensive medication. In another report, greater variability of systolic BP was an independent risk factor associated with frailty status [83]. These results suggest the need to consider the pros and cons of antihypertensive treatment individually to prevent frailty and other geriatric syndromes in older adults.

In a cross-sectional study of the association between cardiovascular risk profile and frailty in the Nambu Cohort Study (mean age 78 years), 37\% of cases of frailty were diagnosed using the Kihon Checklist score (Supplementary Information 7-2). For every $10 \mathrm{mmHg}$ increase in systolic $\mathrm{BP}$, the risk of frailty decreased significantly by $17 \%$. Moreover, the total number of cardiovascular risk profiles within the optimal range (systolic $\mathrm{BP}<140 / 90 \mathrm{mmHg}$, LDL-cholesterol $<100 \mathrm{mg} / \mathrm{dL}$ and $\mathrm{BMI}<25 \mathrm{~kg} / \mathrm{m}^{2}$ ) was significantly associated with the risk of frailty. These results suggest that a favorable cardiovascular risk profile, called "reverse metabolic syndrome", might be a risk for frailty.

Sarcopenia is an essential component of frailty, and chronic diseases, including CVD, are a common cause of sarcopenia. However, there has been little clinical evidence on the mechanism of sarcopenia in patients with CVD. A retrospective cross-sectional study in the Kyushu area (mean age 72 years) showed an association among skeletal muscle mass reduction, endothelial function, and markers of advanced vascular damage in older patients with CVD (Supplementary Information 7-3). Of cases of sarcopenia, $25.5 \%$ were diagnosed using the Asian Working Group for 


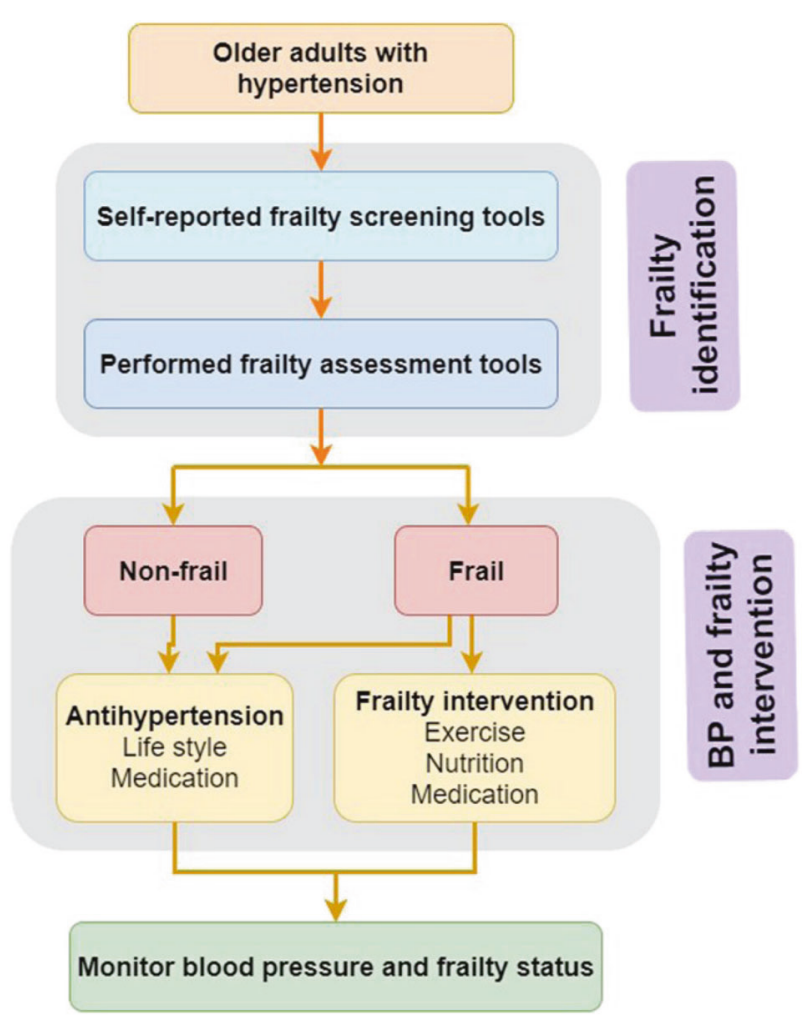

Fig. 7 Proposed management process for hypertension in frail older adults. Cited from ref. [84]

Sarcopenia criteria. Greater progression of arterial stiffness, shown by a higher arterial velocity pulse index (AVI), and more severe tissue damage, shown by a narrower bioelectrical phase angle (PA), were found in subjects with sarcopenia. Both AVI and PA, as well as gender, age, and presence of hypertension, were independently correlated with skeletal muscle index. These results suggest that advanced vascular damage, called "vascular aging", may contribute to the development of sarcopenia, possibly through skeletal muscle tissue damage in patients with CVD.

The risk of development of CVD, such as elevated systolic BP or BP variability, high LDL-cholesterol, and obesity, should be controlled within the optimal range in nonolder or robust older adults; however, in frail older adults, a favorable cardiovascular risk profile may act as an adverse prognostic factor, and in addition, advanced vascular damage is thought to be associated with sarcopenia. Therefore, especially in older patients with a long duration of hypertension or CVD, it is important to keep in mind that strict control of risk factors may lead to a poor prognosis through the progression of frailty. Therefore, it is strongly recommended to consider whether to prioritize antihypertensive treatment or frailty intervention based on the evaluation of frailty status (Fig. 7) [84].

\section{New chemiluminescent enzyme immunoassays for aldosterone measurement and nonsteroidal mineralocorticoid receptor antagonists in the diagnosis and management of primary aldosteronism}

\section{(See Supplementary Information 8)}

During 2020-2021, large numbers of papers regarding primary aldosteronism have been published in Hypertension Research and other journals. It is recommended that the diagnosis of primary aldosteronism (PA) is performed in a stepwise manner; screening, confirmatory testing and subtype testing. The biggest topic in Japan is the discontinuation of the previous radioimmunoassay for aldosterone measurement in March 2021, and the launch of alternative new chemiluminescent enzyme immunoassays (CLEIA) for aldosterone measurement. Nishikawa et al. [85], Ozeki et al. [86], and Teruyama et al. [87] demonstrated that the new CLEIA-plasma aldosterone concentration (PAC) is almost equal to the value obtained by LC-MS/MS; however, the CLEIA-PAC value was shown to be lower than the old RIA-PAC value. Therefore, these changes markedly affect PA diagnosis. The PA clinical practice guidelines 2021 will soon be published by the Japan Endocrine Society. When saline infusion test (SIT) is performed for confirmatory testing, Yamashita et al. (Supplementary Information 8-1) showed that shortened SIT (1 L saline loading over $2 \mathrm{~h}$ ) may be as useful as the standard SIT ( $2 \mathrm{~L}$ saline loading over $4 \mathrm{~h}$ ) with a cutoff value of $66 \mathrm{pg} / \mathrm{mL}$. In adrenal vein sampling, use of cosyntropin stimulation is controversial; however, Yatabe et al. (Supplementary Information 8-2) showed that it improved the judgment of successful adrenal vein catheterization and outcome prediction. Nishimoto et al. [88] showed that immunohistochemical staining for aldosterone synthase revealed diverse PA pathology, and hypokalemia $(<2.82 \mathrm{mEq} / \mathrm{L})$ may independently predict a small PA lesion. PA is a clinically important disease because it is associated with a high prevalence of cardiovascular complications, such as abdominal aortic calcification, skin microvascular dysfunction, and microalbuminuria, as shown by Tuersun et al. (Supplementary Information 8-3), Concistre et al. (Supplementary Information 8-4), and Wang et al. (Supplementary Information 8-5), respectively.

The next hot topic is the development of novel nonsteroidal mineralocorticoid receptor (MR) antagonists, esaxerenone and finerenone. Both MR antagonists showed high affinity as well as high selectivity for MR. Esaxerenone was approved in Japan in January 2019 for the treatment of hypertension. Ito et al. [89] showed that esaxerenone had sufficient antihypertensive effects and was well tolerated in hypertensive patients with moderate kidney dysfunction (eGFR $30-60 \mathrm{~mL} / \mathrm{min} / 1.73 \mathrm{~m}^{2}$ ), as both monotherapy and add-on therapy to a RAS inhibitor. Satoh 


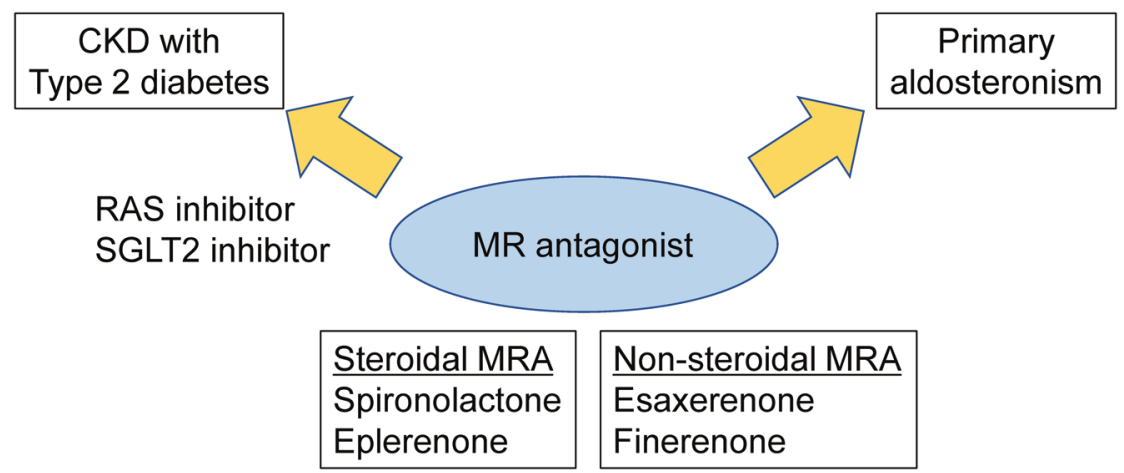

Fig. 8 Role of MR antagonists in primary aldosteronism and CKD with and without diabetes. Esaxerenone and finerenone have been developed as novel nonsteroidal MR antagonists. It is crucial to select an appropriate MR antagonist (steroidal or nonsteroidal) according to the disease state. Recent clinical trials showed that the combination of a renin-angiotensin system (RAS) inhibitor and a sodium-glucose cotransporter-2 (SGLT2) inhibitor with an MR antagonist may have benefit for renoprotection as well as cardiovascular protection. Each ref. number indicates the reference paper cited in the text

with risk of target organ damage and worse cardiovascular outcome in the general, hypertensive and high-risk populations, independent of average BP level [96-106]. Generally, BPV has been classified into very short-term, shortterm, mid-term and long-term BPV according to the measurement period. In 2020, Hypertension Research published a paper about BPV in diverse conditions (Fig. 9). In the first study, in acute ischemic stroke patients, high BP variability measured in the supine position every $4 \mathrm{~h}$ during the first 24 $\mathrm{h}$ of admission was associated with an unfavorable outcome within 30 days after onset (Supplementary Information 9-1). Alexandrou et al. compared short-term BPV assessed by $\mathrm{ABP}$ monitoring (ABPM) in peritoneal dialysis (PD) patients with hemodialysis (HD) patients (Supplementary Information 9-2). The results showed that there was no significant difference in short-term BPV between PD and HD patients.

Manousopoulos et al. reported that short-term BPV assessed by ABPM, but not mid-term BPV assessed by HBP monitoring (day-to-day home BPV), was associated with left ventricular mass index in CKD patients defined by eGFR $<60 \mathrm{ml} / \mathrm{min} / 1.73 \mathrm{~m}^{2}$ [107]. This result showed a discrepancy with a previous study [108]. The reason may be due to differences in the populations. The prevalence of diabetes was $53.3 \%$ and $9 \%$ in the present and previous study, respectively. For example, we previously reported that increased day-to-day home BPV was associated with reduced eGFR in individuals with diabetes, but not in those without [109]. Although increased day-to-day home BPV was associated with the risk of cardiovascular events independent of average home BP level [96], further study is needed to determine whether the clinical impact of day-today home BPV is different in high-risk populations such as individuals with diabetes or CKD or both.

Liu et al. reported that in pregnancy, increased long-term BPV assessed by OBP measured from 20 weeks of 


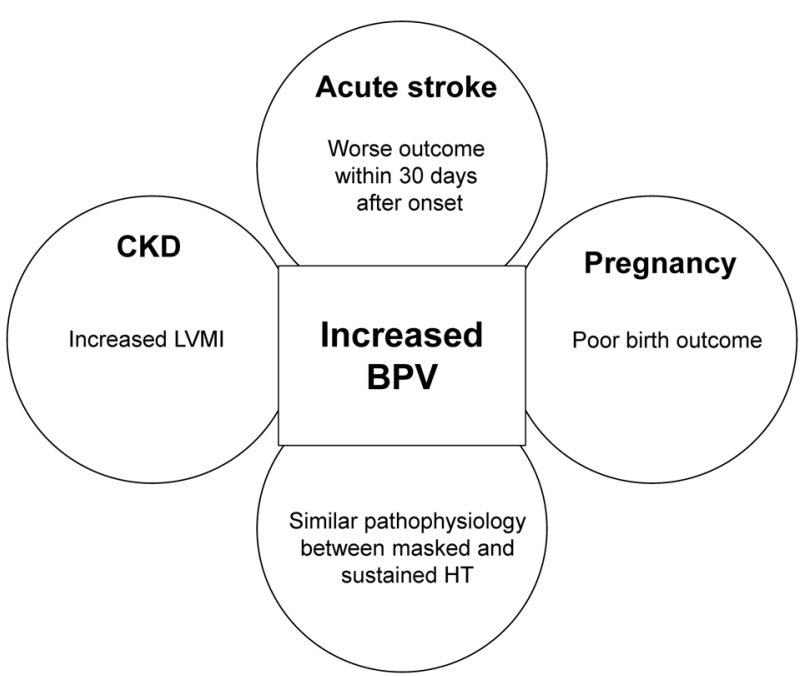

Fig. 9 Topics on blood pressure variability. BPV blood pressure variability, CKD chronic kidney disease, HT hypertension

gestation onwards was associated with poor birth outcomes [110]. On the other hand, the association between the maternal HBP trajectory and lower infant birth weight showed a J-curve relationship [111]. Thus, even in pregnancy, management using office and out-of-office BP measurement is important.

Another topic is whether masked and white-coat hypertension, which are caused by BP variability between office and out-office BP measurements, have different pathological mechanisms, from the viewpoint of BPV, from sustained hypertension. Sheng et al. reported that masked, but not white-coat hypertension, showed a similar pattern of change in BP and heart rate variability and baroreceptor sensitivity assessed with a Finometer device to those in sustained hypertension [80]. This result confirms the previous study results that masked hypertension exerted a risk of poor cardiovascular outcome similarly to sustained hypertension [112]. Although beat-to-beat BP measurement is needed to estimate BPV accurately and reliably, there is no validated and simplified device that is available to use in clinical settings. In the future, the development of BP monitoring is expected in this field [113, 114].

\section{Uric acid}

(See Supplementary Information 10)

Serum uric acid level is regulated by renal excretion, extrarenal excretion in the gut and de novo synthesis in the liver. These are affected by the genetic predisposition for uric acid transporters (kidney and gut) and also by environmental factors. Hyperuricemia is the cause of gout and is also closely associated with the development of hypertension (Fig. 10) [115, 116]. The mechanism by which uric

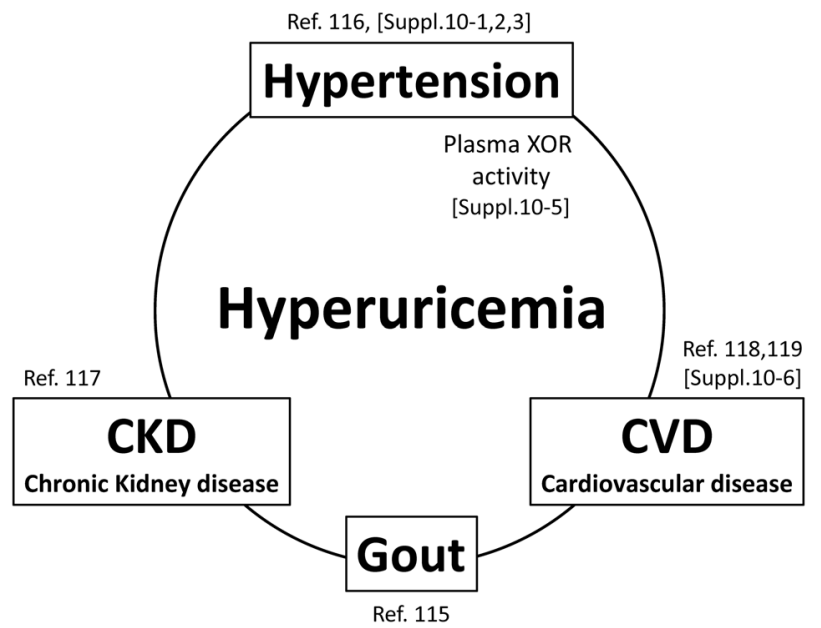

Fig. 10 Association of hyperuricemia with hypertension, gout, chronic kidney disease (CKD) and cardiovascular disease (CVD). Hyperuricemia is known to be the etiological mechanism of gout. In recent papers in Hypertension Research, elevated serum uric acid level was reported to be a risk factor for subsequent development of hypertension. Furthermore, elevated serum uric acid level has been reported to be associated with chronic kidney disease (CKD), and also potentially with cardiovascular disease (CVD). Each ref. number indicates the reference paper cited in the text

acid is involved in hypertension is assumed to be endothelial dysfunction related to the crystal pathway (extracellular uric acid) and crystal-independent pathway (intracellular uric acid) [116].

Recently, a cross-sectional study in Japanese subjects ( $n=236,22)$ who underwent heath checkups demonstrated a J-shaped association between serum uric acid level and the prevalence of BP abnormalities (Supplementary Information 10-1). It was also reported in Hypertension Research that hyperuricemia was a risk for developing hypertension independently of alcohol consumption (Saku study) (Supplementary Information 10-2), and that elevated serum uric acid level in subjects aged 12-13 years was associated with elevated BP in young adults (Fig. 10) (Supplementary Information 10-3). Some SNPs have been reported to be involved in the increase in serum uric acid by thiazide-like diuretics (Supplementary Information 10-4). Taking these findings together, including those in previous papers, it is well established that elevated serum uric acid level is one of the risk factors for the subsequent development of hypertension. On the other hand, elevated plasma activity of xanthine oxidoreductase (XOR) was reported to be associated with hypertension independently of uric acid level (Supplementary Information 10-5). Since patients with hyperuricemia comprise an etiologically heterogeneous population, there may be differences in its complications such as hypertension, depending on the subpopulation. 


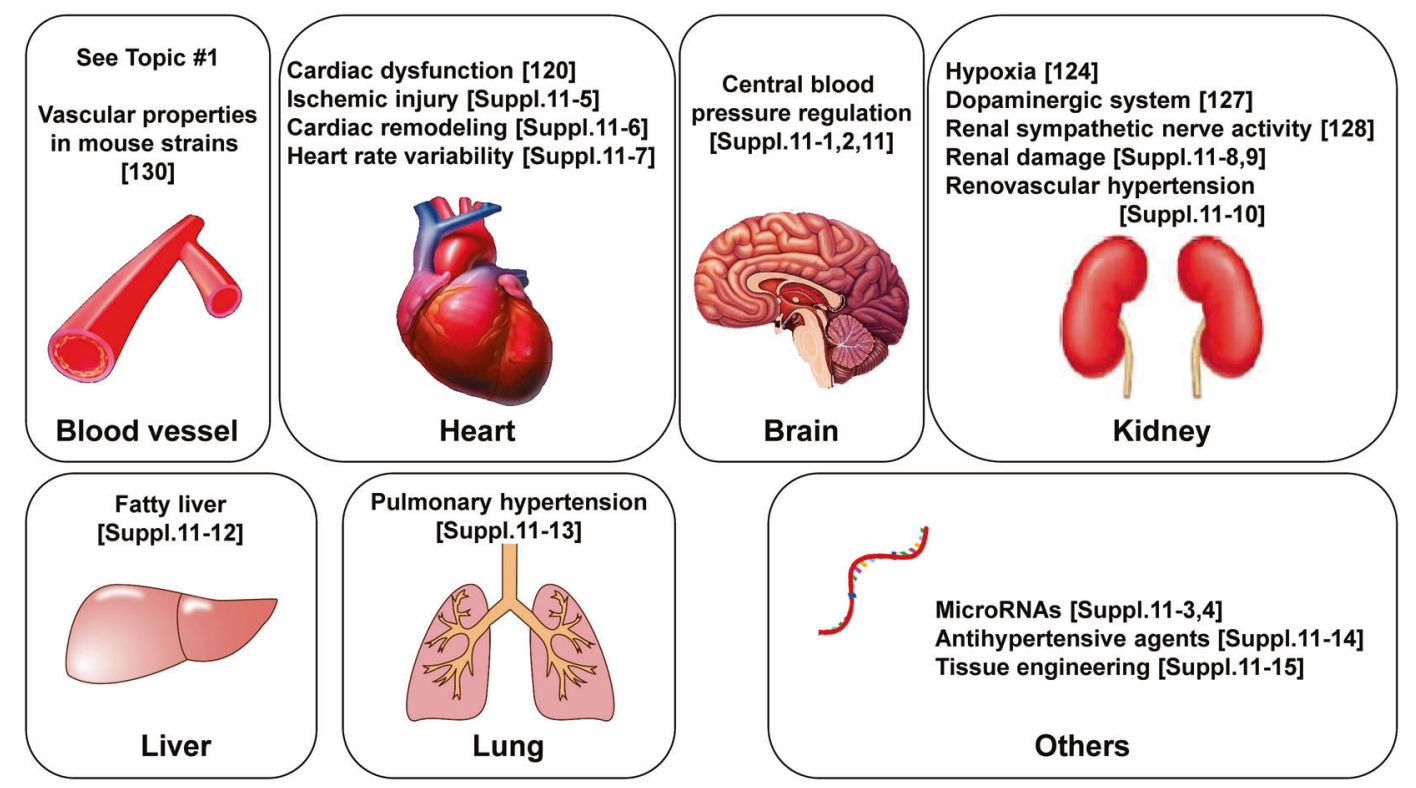

Fig. 11 Topics in basic research. Each ref. number indicates the reference paper cited in the text

In Hypertension Research 2020, papers on the association of uric acid with CKD and CVD from cohort studies in the Japanese general population such as BOREAS-CKD2, CIRCS, and the Hisayama Study were published (Fig. 10) [117-119]. Elevated serum uric acid level was associated with a decline in renal function [117], was an independent predictor of total strokes in women [118], and was a risk for cardiovascular mortality [119]. The causality between serum uric acid and stroke was taken up as a topic in Hypertension Research (Supplementary Information 10-6). Besides, serum uric acid level was high in adult patients with congenital heart disease showing high brachial-ankle pause wave velocity (baPWV) (Supplementary Information 10-7). Basic studies are needed to elucidate the mechanism (s) of uric acid not only in hypertension but also in atherosclerosis. Accumulated evidence from epidemiological studies suggests that hyperuricemia is also a risk factor for CKD and CVD. However, the clinical significance of intervention for serum uric acid in CKD and CVD remains controversial. Further progress in clinical research is expected in the near future.

\section{Progress in translational research: basic research}

\section{(See Supplementary Information 11)}

Basic research plays important roles in the progress of translational research (Fig. 11). First, in the heart, Peterson et al. demonstrated the enhancing effects of caspase recruitment domain family member 9 (Card9) on cardiac fibrosis and hypertrophy using a transverse aortic constriction model [120]. The authors previously reported a protective effect against ischemia and reperfusion injury through attenuation of acute inflammatory responses in mice without the Card9 gene [121], indicating Card9 to be a potential target in CVD [122]. On the other hand, another Card family member, Card6, is reported to be a novel cardioprotective factor via negative regulation of mitogenactivated protein kinase signaling [123]. "Cards" act as different master cards in cardiac disease.

In the kidney, Natarajan et al. reported the effects of acute hypoxia on renal epithelial cells, with enhanced mitochondrial function [124]. Young spontaneously hypertensive rats (SHR) show increased renal expression of key proteins for mitochondrial biogenesis and hypoxiainducible factor (HIF)-1 $\alpha$ compared to normotensive Wistar Kyoto rats. Increased mitochondrial metabolism could be a causal contributor to hypertension in SHR [125]. On the other hand, HIF- $1 \alpha$ activation improves mitochondrial function in early CKD and has a beneficial effect on renal function, with lowered mitochondrial oxygen consumption [126]. Thus, the roles of HIF-1 $\alpha$ have been focused on in both hypertension and CKD. Moreover, Kouyoumdzian et al. reported the effects of angiotensin II on the renal dopaminergic system [127]. They presented a clear schematic representation of this mechanism, showing the association between dopamine D1-like receptor (D1R) and angiotensin type 1 receptor (AT1R), focusing on the counteraction of the effects of dopamine and angiotensin II on sodium excretion. On the other hand, a role of the D1R and angiotensin type 2 receptor (AT2R) interaction in maintaining sodium ion transport has been reported [126]. Interestingly, AT2R stimulation was regulated by 
angiotensin III. There are marked interactions between dopamine receptors and angiotensin receptors. Morisawa et al. demonstrated the beneficial effects of renal denervation on salt-sensitive hypertension by reducing its effects on catabolism and cardiovascular energy expenditure [128]. Hypertension increases glucose utilization in cardiac muscle prior to the development of structural changes [129]. Renal denervation is expected to have not only BP-lowering effects but also BP-independent cardioprotective effects, with attenuation of the salt-derived catabolic state.

In the brain, Savic et al. (Supplementary Information 11-1) showed possible roles of vasopressin, with an increase in gene expression of vasopressin and $\mathrm{V} 1 \mathrm{~b}$ receptor in the hypothalamic paraventricular nuclei in borderline hypertensive rats. Moreover, Cao et al. (Supplementary Information 11-2) demonstrated that microinjection of urotensin II, a polypeptide molecule with neurohormonelike activity, into the rostral ventrolateral medulla increases sympathetic vasomotor tone. Such vasoactive peptides have also been highlighted in hypertension research to elucidate the central regulation of BP.

Jackson et al. (Supplementary Information 11-3) demonstrated that neural suppression of miRNA-181a in the kidney increases renin expression, resulting in exacerbation of hypertension in BPH/2J mice. Moreover, Zhang et al. (Supplementary Information 11-4) showed that an increased circulating level of miR-122 could be a potential risk factor for endothelial dysfunction in young hypertensive mice. MicroRNAs have been highlighted as future clinical biomarkers and therapeutic tools in hypertension.

Finally, Steppan et al. reported mouse strain-dependent differences in key vascular stiffness indices among inbred mouse strains such as C57BL/6J, 129S, and BL6/129S [130]. These baseline data may contribute to future animal studies in hypertension research. Moreover, much promising basic research has been reported in Hypertension Research. See Supplementary Information.

\section{Compliance with ethical standards}

Conflict of interest The authors declare no competing interests.

Publisher's note Springer Nature remains neutral with regard to jurisdictional claims in published maps and institutional affiliations.

\section{References}

1. Celermajer DS, Sorensen KE, Gooch VM, Spiegelhalter DJ, Miller OI, Sullivan ID, et al. Non-invasive detection of endothelial dysfunction in children and adults at risk of atherosclerosis. Lancet. 1992;340:1111-5.

2. Corretti MC, Anderson TJ, Benjamin EJ, Celermajer D, Charbonneau F, Creager MA, et al. Guidelines for the ultrasound assessment of endothelial-dependent flow-mediated vasodilation of the brachial artery: a report of the International Brachial
Artery Reactivity Task Force. J Am Coll Cardiol. 2002;39:257-65.

3. Higashi Y. Assessment of endothelial function: history, methodological aspects, and clinical perspectives. Int Heart J. 2015;56:125-34.

4. Higashi Y, Noma K, Yoshizumi M, Kihara Y. Oxidative stress and endothelial function in cardiovascular diseases. Circ J. 2009;73:411-8.

5. Maruhashi T, Soga J, Fujimura N, Idei N, Mikami S, et al. Nitroglycerine-induced vasodilation for assessment of vascular function: a comparison with flow-mediated vasodilation. Arterioscler Thromb Vasc Biol. 2013;33:1401-8.

6. Tanaka A, Tomiyama H, Maruhashi T, Matsuzawa Y, Miyoshi T, Kabutoya T, et al. Physiological diagnosis criteria for vascular failure. Hypertension. 2018;72:1060-71.

7. Soga J, Noma K, Hata T, Hidaka T, Fujii Y, Idei N, et al. for ROCK investigator group. Rho-associated kinase (ROCK) activity, endothelial function and cardiovascular risk factors. Arterioscler Thromb Vasc Biol. 2011;31:2353-9.

8. Maruhashi T, Soga J, Idei N, Fujimura N, Mikami S, Iwamoto Y, et al. Relationship between flow-mediated vasodilatation and cardiovascular risk factors in a large community-based study. Heart. 2013;99:1837-42.

9. Fujimura N, Noma K, Hata T, Soga J, Hidaka T, et al. for ROCK investigator group. Selective mineralocorticoid receptor blocker eplerenone improves endothelial function and inhibits Rhoassociated kinase activity in patients with essential hypertension. Clin Pharmacol Ther. 2012;91:289-97.

10. Higashi Y, Sasaki S, Nakagawa K, Matsuura H, Oshima T, Chayama K. Endothelial function and oxidative stress in renovascular hypertension. N Engl J Med. 2002;346:1954-62.

11. Modena MG, Bonetti L, Coppi F, Bursi F, Rossi R. Prognostic role of reversible endothelial dysfunction in hypertensive postmenopausal women. J Am Coll Cardiol. 2002;40:505-10.

12. Lerman A, Zeiher AM. Endothelial function: cardiac events. Circulation. 2005;111:363-8.

13. Liu Z, Yang Y, Zhang Y, Xie L, Li Q, Song Y, et al. Association of brachial-ankle pulse wave velocity and carotid plaque in Chinese hypertensive adults: effect modification by age. Hypertens Res. 2020;43:808-16.

14. Maruhashi T, Soga J, Fujimura N, Idei N, Mikami S, Iwamoto Y, et al. Increased arterial stiffness and cardiovascular risk prediction in controlled hypertensive patients with coronary artery disease: post hoc analysis of the FMD-J (Flow-mediated Dilation Japan) Study A. Hypertens Res. 2020;43:781-90.

15. Bellien J, Iacob M, Richard V, Wils J, Cam-Duchez VL, Joannides R. Evidence for wall shear stress-dependent t-PA release in human conduit arteries: role of endothelial factors and impact of high blood pressure. Hypertens Res. 2020;44:310-7.

16. Maruhashi T, Kajikawa M, Kishimoto S, Hashimoto H, Takaeko $\mathrm{Y}$, Yamaji T, et al. Vascular function is further impaired in subjects aged 80 years of older. Hypertens Res. 2020;43:914-21.

17. Roberts J, Redman C. Pre-eclampsia: more than pregnancyinduced hypertension. Lancet. 1993;341:1447-51.

18. Li N, An H, Li Z, Ye R, Zhang L, Li H, et al. Preconception blood pressure and risk of gestational hypertension and preeclampsia: a large cohort study in China. Hypertens Res. 2020;43:956-62.

19. Goto M, Koide K, Tokunaka M, Takita H, Hamada S, Nakamura M, et al. Accuracy of the FMF Bayes theorem-based model for predicting preeclampsia at 11-13 weeks of gestation in a Japanese population. Hypertens Res. 2021;44:685-91.

20. Huang Q, Liu L, Hu B, Di X, Brennecke SP, Liu H. Decreased seizure threshold in an eclampsia-like model induced in pregnant rats with lipopolysaccharide and pentylenetetrazol treatments. PLoS ONE. 2014;9:1-8. 
21. Wu P, Haththotuwa R, Kwok CS, Babu A, Kotronias RA, Rushton C, et al. Preeclampsia and future cardiovascular health. Circ Cardiovasc Qual Outcomes. 2017;10:1-9.

22. Engeland A, Bjørge T, Daltveit AK, Skurtveit S, Vangen S, Vollset SE, et al. Risk of diabetes after gestational diabetes and preeclampsia. A registry-based study of 230,000 women in Norway. Eur J Epidemiol. 2011;26:157-63.

23. Khashan AS, Evans M, Kublickas M, McCarthy FP, Kenny LC, Stenvinkel P, et al. Erratum: Preeclampsia and risk of end stage kidney disease: a Swedish nationwide cohort study. PLoS Med. 2019;16:e1002875.

24. Basit S, Wohlfahrt J, Boyd HA. Pre-eclampsia and risk of dementia later in life: Nationwide cohort study. BMJ. 2018;363: $\mathrm{k} 4109$.

25. Wagata M, Kogure M, Nakaya N, Tsuchiya N, Nakamura T, Hirata $\mathrm{T}$, et al. Hypertensive disorders of pregnancy, obesity, and hypertension in later life by age group: a cross-sectional analysis. Hypertens Res. 2020;43:1277-83.

26. Shibata S, Arima H, Asayama K, Hoshide S, Ichihara A, Ishimitsu $\mathrm{T}$, et al. Hypertension and related diseases in the era of COVID-19: a report from the Japanese Society of Hypertension Task Force on COVID-19. Hypertens Res. 2020;43:1028-46.

27. Matsuzawa Y, Ogawa H, Kimura K, Konishi M, Kirigaya J, Fukui K, et al. Renin-angiotensin system inhibitors and the severity of coronavirus disease 2019 in Kanagawa, Japan: a retrospective cohort study. Hypertens Res. 2020;43:1257-66.

28. de Abajo FJ, Rodríguez-Martín S, Lerma V, Mejía-Abril G, Aguilar M, García-Luque A, et al. Use of renin-angiotensinaldosterone system inhibitors and risk of COVID-19 requiring admission to hospital: a case-population study. Lancet. 2020;395:1705-14.

29. Mancia G, Rea F, Ludergnani M, Apolone G, Corrao G. Reninangiotensin-aldosterone system blockers and the risk of Covid19. N Engl J Med. 2020;382:2431-40.

30. Sheppard JP, Nicholson BD, Lee J, McGagh D, Sherlock J, Koshiaris C, et al. Association between blood pressure control and coronavirus disease 2019 outcomes in 45418 symptomatic patients with hypertension: an observational cohort study. Hypertension. 2021;77:846-55.

31. Modesti PA, Wang J, Damasceno A, Agyemang C, Van Bortel $\mathrm{L}$, Persu A, et al. Indirect implications of COVID-19 prevention strategies on non-communicable diseases: An Opinion Paper of the European Society of Hypertension Working Group on Hypertension and Cardiovascular Risk Assessment in Subjects Living in or Emigrating from Low Resource Settings. BMC Med. 2020;18:256.

32. Palmer K, Monaco A, Kivipelto M, Onder G, Maggi S, Michel JP, et al. The potential long-term impact of the COVID-19 outbreak on patients with non-communicable diseases in Europe: consequences for healthy ageing. Aging Clin Exp Res. 2020;32:1189-94.

33. Bello B, Useh U. COVID-19: Are non-communicable diseases risk factors for its severity? Am J Health Promot. 2020;35:720-9.

34. Cheung AK, Chang TI, Cushman WC, Furth SL, Hou FF, Ix JH, et al. KDIGO 2021 clinical practice guideline for the management of blood pressure in chronic kidney disease. Kidney Int. 2021;99:S1-87.

35. Wright JT Jr., Williamson JD, Whelton PK, Snyder JK, Sink $\mathrm{KM}$, Rocco MV, et al. A randomized trial of intensive versus standard blood-pressure control. N Engl J Med. 2015;373:2103-16.

36. Yoshimura R, Yamamoto R, Shinzawa M, Tomi R, Ozaki S, Fujii Y, et al. Frequency of alcohol drinking modifies the association between salt intake and albuminuria: a 1-year observational study. Hypertens Res. 2020;43:1249-56.
37. Bakris GL, Agarwal R, Anker SD, Pitt B, Ruilope LM, Rossing $\mathrm{P}$, et al. Effect of finerenone on chronic kidney disease outcomes in type 2 diabetes. N Engl J Med. 2020;383:2219-29.

38. Heerspink HJL, Stefánsson BV, Correa-Rotter R, Chertow GM, Greene T, Hou FF, et al. Dapagliflozin in patients with chronic kidney disease. N Engl J Med. 2020;383:1436-46.

39. Packer M, Anker SD, Butler J, Filippatos G, Pocock SJ, Carson $\mathrm{P}$, et al. Cardiovascular and renal outcomes with empagliflozin in heart failure. N Engl J Med. 2020;383:1413-24.

40. Bhatt DL, Szarek M, Pitt B, Cannon CP, Leiter LA, McGuire DK, et al. Sotagliflozin in patients with diabetes and chronic kidney disease. N Engl J Med. 2021;384:129-39.

41. Heerspink HJL, Sjöström CD, Jongs N, Chertow GM, Kosiborod M, Hou FF, et al. Effects of dapagliflozin on mortality in patients with chronic kidney disease: a pre-specified analysis from the DAPA-CKD randomized controlled trial. Eur Heart J. 2021;42:1216-27.

42. Wheeler DC, Toto RD, Stefánsson BV, Jongs N, Chertow GM, Greene T, et al. A pre-specified analysis of the DAPA-CKD trial demonstrates the effects of dapagliflozin on major adverse kidney events in patients with IgA nephropathy. Kidney Int. 2021;100:215-24.

43. Wheeler DC, Stefánsson BV, Jongs N, Chertow GM, Greene T, Hou FF, et al. Effects of dapagliflozin on major adverse kidney and cardiovascular events in patients with diabetic and nondiabetic chronic kidney disease: a prespecified analysis from the DAPA-CKD trial. Lancet Diabetes Endocrinol. 2021;9:22-31.

44. Li CX, Liang S, Gao L, Liu H. Cardiovascular outcomes associated with SGLT-2 inhibitors versus other glucose-lowering drugs in patients with type 2 diabetes: a real-world systematic review and meta-analysis. PLoS ONE. 2021;16:e0244689.

45. Wilcox CS. Antihypertensive and renal mechanisms of SGLT2 (sodium-glucose linked transporter 2) inhibitors. Hypertension. 2020;75:894-901.

46. Santos-Gallego CG, Requena-Ibanez JA, San Antonio R, Ishikawa K, Watanabe S, Picatoste B, et al. Empagliflozin ameliorates adverse left ventricular remodeling in nondiabetic heart failure by enhancing myocardial energetics. J Am Coll Cardiol. 2019;73:1931-44.

47. Marton A, Kaneko T, Kovalik JP, Yasui A, Nishiyama A, Kitada $\mathrm{K}$, et al. Organ protection by SGLT2 inhibitors: role of metabolic energy and water conservation. Nat Rev Nephrol. 2021;17:65-77.

48. Herat LY, Magno AL, Rudnicka C, Hricova J, Carnagarin R, Ward NC, et al. SGLT2 Inhibitor induced sympathoinhibition. JACC Basic Transl Sci. 2020;5:169-79.

49. Wan N, Fujisawa Y, Kobara H, Masaki T, Nakano D, Rahman A, et al. Effects of an SGLT2 inhibitor on the salt sensitivity of blood pressure and sympathetic nerve activity in a nondiabetic rat model of chronic kidney disease. Hypertens Res. 2020;43:492-9.

50. Tian B, Deng Y, Cai Y, Han M, Xu G. Efficacy and safety of combination therapy with sodium-glucose transporter 2 inhibitors and renin-angiotensin system blockers in patients with type 2 diabetes: a systematic review and meta-analysis. Nephrol Dial Transplant. 2021. https://doi.org/10.1093/ndt/gfab048.

51. Kitamura K, Hayashi K, Ito S, Hoshina Y, Sakai M, Yoshino K, et al. Effects of SGLT2 inhibitors on eGFR in type 2 diabetic patients - the role of antidiabetic and antihypertensive medications. Hypertens Res. 2021;44:508-17.

52. Stefan N, Birkenfeld AL, Schulze MB, Ludwig DS. Obesity and impaired metabolic health in patients with COVID-19. Nat Rev Endocrinol. 2020;16:341-2.

53. Costa FF, Rosário WR, Ribeiro Farias AC, de Souza RG, Duarte Gondim RS, Barroso WA. Metabolic syndrome and COVID-19: 
An update on the associated comorbidities and proposed therapies. Diabetes Metab Syndr. 2020;14:809-14.

54. Patel KHK, Li X, Quint JK, Ware JS, Peters NS, Ng FS. Increasing adiposity and the presence of cardiometabolic morbidity is associated with increased Covid-19-related mortality: results from the UK Biobank. BMC Endocr Disord. 2021;21:144.

55. Clark T, Morey R, Jones MD, Marcos L, Ristov M, Ram A, et al. High-intensity interval training for reducing blood pressure: a randomized trial vs. moderate-intensity continuous training in males with overweight or obesity. Hypertens Res. 2020;43:396-403.

56. Nishigaki D, Yamamoto R, Shinzawa M, Kimura Y, Fujii Y, Aoki K, et al. Body mass index modifies the association between frequency of alcohol consumption and incidence of hypertension in men but not in women: a retrospective cohort study. Hypertens Re.s 2020;43:322-30.

57. Kawano Y. Physio-pathological effects of alcohol on the cardiovascular system: its role in hypertension and cardiovascular disease. Hypertens Res. 2010;33:181-91.

58. GBD 2016 Alcohol Collaborators. Alcohol use and burden for 195 countries and territories, 1990-2016: a systematic analysis for the Global Burden of Disease Study 2016. Lancet. 2018;392:1015-35.

59. Marcos A, Serra-Majem L, Pérez-Jiménez F, Pascual V, Tinahones FJ, Estruch R. Moderate consumption of beer and its effects on cardiovascular and metabolic health: an updated review of recent scientific evidence. Nutrients. 2021;13:879.

60. Imai Y, Hirata T, Saitoh S, Ninomiya T, Miyamoto Y, Ohnishi $\mathrm{H}$, et al. Impact of hypertension stratified by diabetes on the lifetime risk of cardiovascular disease mortality in Japan: a pooled analysis of data from the Evidence for Cardiovascular Prevention from Observational Cohorts in Japan study. Hypertens Res. 2020;43:1437-44.

61. Ushigome E, Kitagawa N, Kitagawa N, Tanaka T, Hasegawa G, Ohnishi M, et al. Predictive power of home blood pressure measurement for cardiovascular outcomes in patients with type 2 diabetes: KAMOGAWA-HBP study. Hypertens Res. 2021;44:348-54.

62. Emdin CA, Rahimi K, Neal B, Callender T, Perkovic V, Patel A. Blood pressure lowering in type 2 diabetes: a systematic review and meta-analysis. JAMA. 2015;313:603-15.

63. Bangalore S, Kumar S, Lobach I, Messerli FH. Blood pressure targets in subjects with type 2 diabetes mellitus/impaired fasting glucose: observations from traditional and bayesian randomeffects meta-analyses of randomized trials. Circulation. 2011;123:2799-810.

64. Reboldi G, Gentile G, Angeli F, Ambrosio G, Mancia G, Verdecchia P. Effects of intensive blood pressure reduction on myocardial infarction and stroke in diabetes: a meta-analysis in 73,913 patients. J Hypertens. 2011;29:1253-69.

65. Ueki K, Sasako T, Okazaki Y, Kato M, Okahata S, Katsuyama $\mathrm{H}$, et al. Effect of an intensified multifactorial intervention on cardiovascular outcomes and mortality in type 2 diabetes ( $\mathrm{J}$ DOIT3): an open-label, randomised controlled trial. Lancet Diabetes Endocrinol. 2017;5:951-64.

66. Umemura S, Arima H, Arima S, Asayama K, Dohi Y, Hirooka Y, et al. The Japanese Society of Hypertension Guidelines for the Management of Hypertension (JSH 2019). Hypertens Res. 2019;42:1235-481.

67. Kai H, Katoh A, Harada H, Niiyama H, Furukawa Y, Kimura T. CREDO-Kyoto Investigators. Low blood pressure and cardiovascular events in diabetic patients with coronary artery disease after revascularization: the CREDO-Kyoto registry cohort-1. Hypertens Res. 2020;43:715-23.
68. Gelžinský J, Mayer O Jr, Seidlerová J, Mateřánková M, Mareš Š, Kordíkova V, et al. Serum biomarkers, skin autofluorescence and other methods. Which parameter better illustrates the relationship between advanced glycation end products and arterial stiffness in the general population? Hypertens Res. 2021;44:518-27.

69. Tanaka A, Tomiyama H, Maruhashi T, Matsuzawa Y, Miyoshi T, Kabutoya T, et al. Physiological diagnostic criteria for vascular failure. Hypertension. 2018;72:1060-71.

70. Hirao J, Tojo A, Hatakeyama S, Satonaka H, Ishimitsu T. V-ATPase blockade reduces renal gluconeogenesis and improves insulin secretion in type 2 diabetic rats. Hypertens Res. 2020;43:1079-88.

71. Grubić Rotkvić P, Planinić Z, Liberati Pršo AM, Šikić J, Galić E, Rotkvić L. The mystery of diabetic cardiomyopathy: from early concepts and underlying mechanisms to novel therapeutic possibilities. Int J Mol Sci. 2021;22:5973.

72. Wang P, Wang D, Yang Y, Hou J, Wan J, Ran F, et al. Tom70 protects against diabetic cardiomyopathy through its antioxidant and antiapoptotic properties. Hypertens Res. 2020;43:1047-56.

73. Sakima A, Satonaka H, Nishida N, Yatsu K, Arima H. Optimal blood pressure targets for patients with hypertension: a systematic review and meta-analysis. Hypertens Res. 2019;42:483-95.

74. Shimbo D, Artinian NT, Basile JN, Krakoff LR, Margolis KL, Rakotz MK, et al. Self-measured blood pressure monitoring at home: a joint policy statement from the American Heart Association and American Medical Association. Circulation. 2020;142:e42-63.

75. Cohen JB, Lotito MJ, Trivedi UK, Denker MG, Cohen DL, Townsend RR. Cardiovascular events and mortality in white coat hypertension: a systematic review and meta-analysis. Ann Intern Med. 2019;170:853-62.

76. Bobrie G, Clerson P, Menard J, Postel-Vinay N, Chatellier G, Plouin PF. Masked hypertension: a systematic review. J Hypertens. 2008;26:1715-25.

77. Uhlig K, Patel K, Ip S, Kitsios GD, Balk EM. Self-measured blood pressure monitoring in the management of hypertension: a systematic review and meta-analysis. Ann Intern Med. 2013;159:185-94.

78. Kadowaki S, Kadowaki T, Hozawa A, Fujiyoshi A, Hisamatsu $\mathrm{T}$, Satoh A, et al. Differences between home blood pressure and strictly measured office blood pressure and their determinants in Japanese men. Hypertens Res. 2021;44:80-7.

79. Gkaliagkousi E, Protogerou AD, Argyris AA, Koletsos N, Triantafyllou A, Anyfanti P, et al. Contribution of single office aortic systolic blood pressure measurements to the detection of masked hypertension: data from two separate cohorts. Hypertens Res. 2021;44:215-24.

80. Sheng CS, Li FK, Cheng YB, Wei FF, Huang JF, Guo QH, et al. Blood pressure and heart rate variability and baroreflex sensitivity in white-coat, masked, and sustained hypertension. Hypertens Res. 2020;43:772-80.

81. Zhang D, Huang QF, Li Y, Wang JG. A randomized controlled trial on home blood pressure monitoring and quality of care in stage 2 and 3 hypertension. Hypertens Res. 2021;44:533-40.

82. Benetos A, Petrovic M, Strandberg T. Hypertension management in older and frail older patients. Circ Res. 2019;124:1045-60.

83. Zhu Y, Chen X, Geng S, Li Q, Yuan H, Zhou X, et al. Association between ambulatory blood pressure variability and frailty among older hypertensive patients. J Clin Hypertens. 2020;22:1703-12.

84. Liu P, Li Y, Zhang Y, Mesbah SE, Ji T, Ma L. Frailty and hypertension in older adults: current understanding and future perspectives. Hypertens Res. 2020;43:1352-60.

85. Nishikawa T, Satoh F, Takashi Y, Yanase Y, Itoh H, Kurihara I, et al. Comparison and commutability study between standardized 
liquid chromatography-mass spectrometry/mass spectrometry (LC-MS/MS) and chemiluminescent enzyme immunoassay for aldosterone measurement in blood. Endocr J. 2021. https://doi. org/10.1507/endocrj.EJ21-0278.

86. Ozeki Y, Tanimura Y, Nagai S, Nomura T, Kinoshita M, Shibuta $\mathrm{K}$, et al. Development of a new chemiluminescent enzyme immunoassay using a two-step sandwich method for measuring aldosterone concentrations. Diagnostics. 2021;11:433.

87. Teruyama K, Naruse M, Tsuiki M, Kobayashi H. Novel chemiluminescent immunoassay to measure plasma aldosterone and plasma active renin concentrations for the diagnosis of primary aldosteronism. J Hum Hypertens. 2021. https://doi.org/10.1038/ s41371-020-00465-5.

88. Nishimoto K, Umakoshi H, Seki T, Yasuda M, Araki R, Otsuki $\mathrm{M}$, et al. Diverse pathological lesions of primary aldosteronism and their clinical significance. Hypertens Res. 2021;44:498-507.

89. Ito $\mathrm{S}$, Itoh $\mathrm{H}$, Rakugi $\mathrm{H}$, Okuda $\mathrm{Y}$, Iijima S. Antihypertensive effects and safety of esaxerenone in patients with moderate kidney dysfunction. Hypertens Res. 2021;44:489-97.

90. Satoh F, Ito S, Itoh H, Rakugi H, Shibata H, Ichihara A, et al. Efficacy and safety of esaxerenone (CS-3150), a newly available nonsteroidal mineralocorticoid receptor blocker, in hypertensive patients with primary aldosteronism. Hypertens Res. 2021;44:464-72.

91. Arai K, Morikawa Y, Ubukata N, Sugimoto K. Synergistic reduction in albuminuria in type 2 diabetic mice by esaxerenone (CS-3150), a novel nonsteroidal selective mineralocorticoid receptor blocker, combined with an angiotensin II receptor blocker. Hypertens Res. 2020;43:1204-13.

92. Ito S, Kashihara N, Shikata K, Nangaku M, Wada T, Okuda Y, et al. Esaxerenone (CS-3150) in patients with type 2 diabetes and microalbuminuria (ESAX-DN): Phase 3 randomized controlled clinical trial. Clin J Am Soc Nephrol. 2020;15:1715-27.

93. Bakris GL, Agarwal R, Anker SD, Pitt B, Ruilope LM, Rossing $\mathrm{P}$, et al. Effect of finerenone on chronic kidney disease outcomes in type 2 diabetes. N Engl J Med. 2020;383:2219-29.

94. Sarafidis P, Papadopoulos CE, Kamperidis V, Giannakoulas G, Doumas M. Cardiovascular protection with sodium-glucose cotransporter-2 inhibitors and mineralocorticoid receptor antagonists in chronic kidney disease. A milestone achieved. Hypertension. 2021;77:1442-55.

95. Yokota K, Shibata H, Kurihara I, Kobayashi S, Murai-Takeda A, Itoh $\mathrm{H}$. CASZ1b is a novel transcriptional corepressor of mineralocorticoid receptor. Hypertens Res. 2021;44:407-16.

96. Hoshide S, Yano Y, Mizuno H, Kanegae H, Kario K. Day-by-day variability of home blood pressure and incident cardiovascular disease in clinical practice: The J-HOP study (Japan Morning SurgeHome Blood Pressure). Hypertension. 2018;71:177-84.

97. Rothwell PM, Howard SC, Dolan E, O'Brien E, Dobson JE, Dahlöf $B$, et al. Prognostic significance of visit-to-visit variability, maximum systolic blood pressure, and episodic hypertension. Lancet. 2010;375:895-905.

98. Asayama K, Kikuya M, Schutte R, Thijs L, Hosaka M, Satoh M, et al. Home blood pressure variability as cardiovascular risk factor in the population of Ohasama. Hypertension. 2013;61:61-9.

99. Ishiyama Y, Hoshide S, Kanegae H, Kario K. Increased arterial stiffness amplifies the association between home blood pressure variability and cardiac overload: The J-HOP study. Hypertension. 2020;75:1600-6.

100. Chia YC, Kario K, Tomitani N, Park S, Shin J, Turana Y, et al. Comparison of day-to-day blood pressure variability in hypertensive patients with type 2 diabetes mellitus to those without diabetes: Asia BP@Home Study. J Clin Hypertens. 2020;22:407-14.
101. Cho N, Hoshide S, Nishizawa M, Fujiwara T, Kario K. Relationship between blood pressure variability and cognitive function in elderly patients with good blood pressure control. Am J Hypertens. 2018;31:293-8.

102. Hoshide S, Yano Y, Shimizu M, Eguchi K, Ishikawa J, Kario K. Is home blood pressure variability itself an interventional target beyond lowering mean home blood pressure during antihypertensive treatment? Hypertens Res. 2012;35:862-6.

103. Takahari K, Nagai M. Higher visit-to-visit blood pressure variability and n-terminal pro-brain natriuretic peptide elevation: Influence of left ventricular hypertrophy and left ventricular diastolic function. Blood Press Monit. 2020;25:126-30.

104. Farrag HMA, Amin AS, Abdel-Rheim AR. Relation of shortterm blood pressure variability to early renal effects in hypertensive patients with controlled blood pressure. Blood Press Monit. 2019;24:221-4.

105. Cuspidi C, Carugo S, Tadic M. Blood pressure variability and target organ damage regression in hypertension. J Clin Hypertens. 2021;23:1159-61.

106. Zhang Y, Bie L, Li M, Wang T, Xu M, Lu J, et al. Visit-to-visit blood pressure variability is associated with arterial stiffness in Chinese adults: a prospective analysis. J Clin Hypertens. 2021;23:802-12.

107. Manousopoulos K, Koroboki E, Barlas G, Lykka A, Tsoutsoura N, Flessa K, et al. Association of home and ambulatory blood pressure variability with left ventricular mass index in chronic kidney disease patients. Hypertens Res. 2021;44:55-62.

108. Matsui Y, Ishikawa J, Eguchi K, Shibasaki S, Shimada K, Kario K. Maximum value of home blood pressure: a novel indicator of target organ damage in hypertension. Hypertension. 2011;57:1087-93.

109. Suzuki D, Hoshide S, Kario K. Associations between day-by-day home blood pressure variability and renal function and albuminuria in patients with and without diabetes. Am J Hypertens. 2020;33:860-8.

110. Liu J, Yang L, Teng H, Cao Y, Wang J, Han B, et al. Visit-tovisit blood pressure variability and risk of adverse birth outcomes in pregnancies in east China. Hypertens Res. 2021;44:239-49.

111. Iwama N, Oba MS, Satoh M, Ohkubo T, Ishikuro M, Obara T, et al. Association of maternal home blood pressure trajectory during pregnancy with infant birth weight: The BOSHI study. Hypertens Res. 2020;43:550-9.

112. Fujiwara T, Yano Y, Hoshide S, Kanegae H, Kario K. Association of cardiovascular outcomes with masked hypertension defined by home blood pressure monitoring in a Japanese general practice population. JAMA Cardiol. 2018;3:583-90.

113. Papaioannou TG, Protogerou AD, Stamatelopoulos KS, Alexandraki KI, Vrachatis D, Argyris A, et al. Very-short-term blood pressure variability: Complexities and challenges. Blood Press Monit. 2020;25:300.

114. Kario K. Management of hypertension in the digital era: Small wearable monitoring devices for remote blood pressure monitoring. Hypertension. 2020;76:640-50.

115. Dalbeth N, Gosling AL, Gaffo A, Abhishek A. Gout. Lancet. 2021;397:1843-55.

116. Lanaspa MA, Andres-Hernando A, Kuwabara M. Uric acid and hypertension. Hypertens Res. 2020;43:832-34.

117. Mori K, Furuhashi M, Tanaka M, Numata K, Hisasue T, Hanawa $\mathrm{N}$, et al. U-shaped relationship between serum uric acid level and decline in renal function during a 10-year period in female subjects: BOREAS-CKD2. Hypertens Res. 2021;44:107-16.

118. Li J, Muraki I, Imano H, Cui R, Yamagishi K, Umesawa M, et al. Serum uric acid and risk of stroke and its types: the Circulatory Risk in Communities Study (CIRCS). Hypertens Res. 2020;43:313-21. 
119. Sakata S, Hata J, Honda T, Hirakawa Y, Oishi E, Shibata M, et al. Serum uric acid levels and cardiovascular mortality in a general Japanese population: the Hisayama Study. Hypertens Res. 2020;43:560-8.

120. Peterson MR, Getiye Y, Bosch L, Sanders AJ, Smith AR, Haller $\mathrm{S}$, et al. A potential role of caspase recruitment domain family member 9 (Card9) in transverse aortic constriction-induced cardiac dysfunction, fibrosis, and hypertrophy. Hypertens Res. 2020;43:1375-84.

121. Qin X, Peterson MR, Haller SE, Cao L, Thomas DP, He G. Caspase recruitment domain-containing protein 9 (CARD9) knockout reduces regional ischemia/reperfusion injury through an attenuated inflammatory response. PLoS ONE. 2018;13:e0199711.

122. Peterson MR, Haller SE, Ren J, Nair S, He G. CARD9 as a potential target in cardiovascular disease. Drug Des Devel Ther. 2016;10:3799-804.

123. Li L, Chen W, Zhu Y, Wang X, Jiang DS, Huang F, et al. Caspase recruitment domain 6 protects against cardiac hypertrophy in response to pressure overload. Hypertension. 2014;64:94-102.

124. Natarajan B, Arige V, Khan AA, Reddy SS, Barthwal MK, Mahapatra NR. Hypoxia-mediated regulation of mitochondrial transcription factors in renal epithelial cells: implications for hypertensive renal physiology. Hypertens Res. 2021;44:154-67.
125. Lee H, Abe Y, Lee I, Shrivastav S, Crusan AP, Huttemann M, et al. Increased mitochondrial activity in renal proximal tubule cells from young spontaneously hypertensive rats. Kidney Int. 2014;85:561-9.

126. Thomas JL, Pham H, Li Y, Hall E, Perkins GA, Ali SS, et al. Hypoxia-inducible factor-1alpha activation improves renal oxygenation and mitochondrial function in early chronic kidney disease. Am J Physiol Ren Physiol. 2017;313:F282-90.

127. Kouyoumdzian NM, Rukavina Mikusic NL, Robbesaul GD, Gorzalczany SB, Carranza A, Trida V, et al. Acute infusion of angiotensin II regulates organic cation transporters function in the kidney: its impact on the renal dopaminergic system and sodium excretion. Hypertens Res. 2021;44:286-98.

128. Morisawa N, Kitada K, Fujisawa Y, Nakano D, Yamazaki D, Kobuchi S, et al. Renal sympathetic nerve activity regulates cardiovascular energy expenditure in rats fed high salt. Hypertens Res. 2020;43:482-91.

129. Polak-Iwaniuk A, Harasim-Symbor E, Golaszewska K, Chabowski A. How hypertension affects heart metabolism. Front Physiol. 2019;10:435.

130. Steppan J, Jandu S, Wang H, Kang S, Savage W, Narayanan R, et al. Commonly used mouse strains have distinct vascular properties. Hypertens Res. 2020;43:1175-81. 